\title{
Une histoire de vocation? Comment les aides-soignantes occultent le processus de transmission de leurs compétences professionnelles
}

\author{
Une enquête France/Québec
}

\author{
François Aubry \\ (Docteur en gérontologie, Université de Franche-Comté \\ et Université de Sherbrooke)
}

\begin{abstract}
Résumé/Abstract
[Fr] Cet article a pour but de montrer comment les aides-soignantes travaillant dans les organisations gériatriques en France et au Québec se représentent le processus par lequel leurs compétences professionnelles leur ont été transmises. Nous avons réalisé vingt-quatre entretiens semi-directifs en France et vingt-trois au Québec (Canada). Nos résultats prouvent que les aides-soignantes utilisent une rhétorique spécifique, celle de la vocation, pour expliquer le sens de leur trajectoire professionnelle. Cet usage rhétorique leur permet de se représenter leur trajectoire dans l'ordre de la continuité. Afin de structurer cette croyance, les aides-soignantes se créent une identité d'endettées, en s'identifiant comme les récipiendaires d'une aide transmise par leurs proches parents, notamment féminins. Elles se disent dans l'obligation morale de rendre ce don aux aînés vivant dans les organisations gériatriques. Ainsi, en utilisant la rhétorique de la vocation, les aides-soignantes françaises tout autant que québécoises occultent le processus de transmission de leurs compétences professionnelles. Ce découpage mémoriel leur permet d'accepter plus facilement les difficultés du métier et de «tenir » face à des situations professionnelles difficiles.

Mots-clefs : aides-soignantes, gériatrie, transmission, compétences professionnelles, mémoire familiale, vocation.

[En] This article aims to show how nurse's aides working in geriatric organizations in France and Quebec represent themselves the process by which professional skills were transmitted to them. We conducted twenty-four semi-structured interviews with nurse's aides in France and twenty-three in Quebec (Canada). Our results show that nurse's aides use a specific rhetoric, that of vocation, to explain the meaning of their professional career. This rhetorical use allows them to represent their path in the order of continuity. In order to structure this belief, nurse's aides create themselves an identity of debt, by identifying themselves as recipients of aid sent by their
\end{abstract}


family, including women. They say that they have a moral obligation to make this gift to seniors living in geriatric organizations. Thus, using the rhetoric of vocation, the nurse's, in France and Quebec, obscure the process of transmission of their skills. The division of memory allows them to more readily accept the challenges of the profession and to "hold" against difficult professional situations.

Key words: nursing assistants, geriatric, transmission, professional skills, family memory, vocation.

\section{Introduction}

L'objectif de cet article est de montrer comment les aides-soignantes ${ }^{1}$ travaillant dans les organisations gériatriques, en France et au Québec, utilisent la notion de vocation pour expliquer le sens de leur trajectoire professionnelle. Cet usage a pour effet d'occulter les mécanismes mêmes par lesquels les compétences professionnelles qu'elles utilisent quotidiennement leur ont été transmises, durant la formation, ou durant des expériences personnelles ou professionnelles spécifiques. La vocation se présente ainsi comme une rhétorique de naturalisation des compétences, à travers laquelle la mémoire de la trajectoire se reconstruit au profit d'une représentation continue et idéelle du parcours professionnel. Cette naturalisation des compétences prouve tout l'intérêt de construire des objets d'études rapprochant les pratiques professionnelles et les représentations mémorielles des acteurs, concernant notamment leur trajectoire biographique.

Les aides-soignantes qui travaillent dans les organisations gériatriques tels que les établissements d'hébergement pour personnes âgées dépendantes (EHPAD) en France et les centres d'hébergement et de soins de longue durée (CHSLD) au Québec sont placées au bas de la hiérarchie organisationnelle. Leur rôle est identique dans les deux contextes, et consiste à réaliser des actes d'assistance envers les personnes âgées dépendantes, ainsi que des actes d'hygiène pour le corps du résident et son environnement immédiat (lit, chambre). Elles détiennent une position centrale dans ces organisations gériatriques, puisqu'elles sont responsables du confort des patients hébergés pour une longue durée. Les compétences qu'elles doivent mettre en œuvre sont donc principalement d'ordre domestique, relatives au caring. Notre étude prend sens précisément dans les contextes éminemment difficiles dans lesquels travaillent ces acteurs professionnels. En effet, les exigences quantitatives (Sainsaulieu 2003) imposées par les directions d'établissements gériatriques publics

\footnotetext{
${ }^{1}$ Nous féminisons le terme «aide-soignante », étant donné la très grande part de femmes dans le métier, soit environ 90\% en France (Arborio 2001) et 80\% au Québec (Grenier 2008). D’autre part, par souci de commodité, nous utilisons le terme « aide-soignante » pour identifier les acteurs professionnels des deux contextes. Mais ces acteurs se nomment "préposés aux bénéficiaires » au Québec.
} 
actuelles, français comme québécois, provoquent des situations complexes que ces acteurs doivent accepter. Plus précisément, l'intensification du travail et l'accentuation des problèmes physiques (obésité) et cognitifs (maladie d'Alzheimer) des résidents (Clément \& Lavoie 2005) sont quelques exemples de la nouvelle donne organisationnelle. En conséquence, le nombre de démissions d'aidessoignantes est important autant en France qu'au Québec, tout comme le nombre de journées d'absence, notamment parmi les jeunes recrues avant leur première année de travail dans l'organisation (Grenier 2008). C'est ainsi que le mode d'intégration des jeunes recrues à l'organisation et le mode de transmission des compétences sont devenus des sujets d'étude prioritaires (Aubry 2010).

Mais l'amélioration possible de la formation de ce personnel se heurte à certaines contradictions. En effet, nous avons constaté que les aides-soignantes des deux contextes ne se représentent pas la transmission de leurs compétences comme une accumulation qui résulte de leur formation ou de leur expérience professionnelle. Elles expliquent plutôt la possession de ces compétences par la vocation qu'elles détiendraient depuis toujours à effectuer le métier. Il est important de questionner cette rhétorique professionnelle afin de comprendre comment ces acteurs transforment leur représentation mémorielle de leur trajectoire, et quelle est la signification de ce processus d'uniformisation de leur parcours professionnel. Nous verrons que la difficulté du travail subie par les aides-soignantes est un élément d'explication fondamental du processus de naturalisation des compétences. Cette fixation sur le caractère naturel de faits éminemment sociaux comme ceux du choix professionnel et de la transmission des compétences a été étudiée notamment par la sociologue Marcelle Stroobants (1993). Elle a conçu une théorie de l'habilitation pour expliquer comment l'acquisition des compétences occasionne nécessairement une transformation cognitive de l'acteur par laquelle il ne se souvient plus du processus de transmission de ses compétences. Nous utiliserons certains éléments de cette théorie dans notre propre analyse.

\section{Méthodologie}

Nous présenterons tout d'abord la méthodologie que nous avons utilisée pour atteindre ces résultats. Ensuite, dans une première partie, nous définirons la vocation comme un recours argumentaire et identitaire utilisé par les acteurs pour décrire leur trajectoire ; nous montrerons que l'usage de cette notion n'est pas inhérent au seul fait des aides-soignantes. Dans une seconde partie, nous montrerons que la naturalisation des compétences prend sens dans un processus de positionnement de soi dans une dynamique générationnelle familiale, qui permet à ces acteurs de mieux expliquer leur choix professionnel. Enfin, nous verrons dans une troisième partie que cette naturalisation des compétences permet pour ces acteurs de mieux accepter le caractère dévalorisé des pratiques qu'elles doivent réaliser. 
Nous avons mené une étude qualitative dans deux CHSLD au Québec et dans deux EHPAD en France. Ces établissements sont publics et ont une taille semblable (environ 150 lits). Ils détiennent un même rôle dans les deux contextes, soit l'accueil et l'assistance envers les personnes âgées dépendantes. Nous avons utilisé une méthode qualitative, puisqu'il s'agissait pour nous de mettre à jour le processus d'argumentation des acteurs sur la question de la transmission de leurs compétences professionnelles. Par ailleurs, nous avons opté pour une approche semi-inductive, en nous inspirant de la théorisation ancrée (Glaser \& Strauss 1967). En effet, le but de cette étude était de recueillir les propos des acteurs et de les catégoriser, tout en axant notre questionnement sur le sujet de la transmission des compétences professionnelles et de la représentation de cette transmission par les acteurs.

Notre méthode de collecte de données a été l'entretien semi-directif. Nous avons interrogé vingt-quatre aides-soignantes en France et vingt-trois au Québec. Un guide d'entretien semi-directif avait été élaboré à cette fin. Précisément, deux thématiques ont structuré notre grille d'entretien. La première partie du guide abordait la trajectoire biographique et le parcours de vie des participantes. Nous avons ainsi précisément demandé à ces acteurs de nous décrire le processus par lequel les compétences qu'elles disent détenir pour effectuer le métier leur ont été transmises. La deuxième partie se penchait sur les conditions de leur intégration dans l'organisation, leur pratique professionnelle à long terme dans les CHSLD (description des tâches, relations construites dans la journée de travail), et l'état des conditions de travail qu'elles subissent (énumération des causes de fatigue, etc.).

Lors des périodes de passation des entretiens et, a fortiori, lors de la période de catégorisation et d'analyse des données, nous avons volontairement adopté une attitude d'ouverture afin de faire place aux données émergentes provenant du terrain. Cette manière de procéder était indispensable pour comprendre quels recours argumentaires les préposées utilisent pour expliquer le processus de transmission des compétences professionnelles. Nous utilisons dans le corps du texte des extraits d'entretiens numérotés et anonymes afin d'illustrer notre propos.

\section{La vocation comme recours argumentaire et identitaire}

Les aides-soignantes que nous avons interrogées présentent communément les compétences qu'elles mettent en œuvre dans leur pratique quotidienne comme des habiletés détenues naturellement. Il est intéressant de noter que nous avons distingué ce processus autant dans les propos des aides-soignantes françaises que québécoises. Dans les deux contextes, les acteurs utilisent des propos illustrant la naturalisation des compétences, tels que «j'ai toujours aimé faire ça » ou «j'ai toujours su quoi faire pour m'occuper des personnes âgées ». Le mot «toujours » tend à créer un sen- 
timent de permanence. Ce sentiment se structure d'un point de vue rhétorique par l'usage du terme de « vocation »:

- Pour faire ce métier-là, je dirais que c'est une question de vocation. Il y a ceux qui peuvent et ceux qui ne peuvent pas. Tu ne viens pas en gériatrie comme ça sans savoir que tu aimes ça. Il faut aimer ça avant de venir ici, sans ça, je pense que tu ne resteras pas longtemps. (E2 - France)

- Ça prend la vocation pour rester ici, je veux dire que tu ne peux pas venir ici en te disant : «Bon je n'aime pas trop les personnes âgées mais ... on peut toujours essayer ... » Non, ça te prend la vocation. Il faut que tu te dises que tu es fait pour ça. (E26 - Québec)

La rhétorique de la vocation permet aux acteurs de présenter leur trajectoire biographique comme un parcours continu, en occultant les étapes de transmission des compétences. Les aides-soignantes énoncent avoir choisi ce métier parce qu'elles savaient détenir depuis toujours ces compétences.

Il faut noter que la naturalisation des compétences n'est pas une rhétorique utilisée seulement par les aides-soignantes. Certains travaux portant sur les professions patrimoniales agricoles (Jacques-Jouvenot 1997) démontrent que la naturalisation des compétences passera par un recours argumentatif au «sang ». Ainsi, « avoir ça dans le sang », chez les agriculteurs, constitue une rhétorique de naturalisation qui s'appuie sur un lien biologique, par lequel les compétences se transmettraient familialement, du père vers le fils. Toutefois, ce n'est pas ce que nous avons observé dans notre étude. Nous ne trouvons pas de marque biologique dans la rhétorique des aides-soignantes. Celles-ci tendent plutôt - comme je l'ai déjà dit - à occulter le processus de transmission des compétences. Les aides-soignantes pensent posséder ces compétences depuis «toujours », et la vocation qu'elles disent ressentir prouverait cette possession.

- Moi je pense que les compétences qu'il faut pour travailler correctement auprès des personnes ici, je veux dire sans violence, sans maltraitance, je pense que ces compétences je les ai en moi, et je pense que je les ai toujours eu. C'est vrai qu'on a une formation mais ce n'est pas cela qui me paraît central, on apprend rien de vraiment important. (E4 - France)

La rhétorique de la vocation a également fait l'objet de recherches dans deux autres domaines de la vie professionnelle, en l'occurrence la religion et l'art. Dans le domaine de la religion, et principalement dans le cas des séminaristes, la vocation passe comme l'instrument subjectif d'explication de la destinée sociale. Pour reprendre Suaud $(1975: 2)$ :

«Qu'elle apparaisse ou qu'elle soit vécue comme un mouvement purement intérieur, comme un acte d'absolue liberté, fait partie intégrante de la vocation ; une vocation « intéressée » ou « forcée » n'est plus, à la limite, une vocation. »

Chez les aides-soignantes, la notion de vocation comporte différentes significations. Reconstruire une perception propre de sa trajectoire professionnelle dans l'ordre de la continuité dispose de certains avantages. Il s'agit tout d'abord d'une 
norme de présentation de soi qui permet de placer une frontière entre celles qui possèderaient les compétences professionnelles nécessaires et celles qui ne les possèderaient pas. Et la sociologue Arborio (2001) a montré en quoi le principe de vocation exprimé par les aides-soignantes recouvre en particulier la partie valorisée du métier, à savoir son aspect relationnel (ou caring). C'est ce segment du métier qui est considéré par les acteurs comme l'objet et la raison de leur vocation. L'usage de ce terme permet alors aux aides-soignantes de se définir comme les détentrices de compétences que d'autres acteurs (aidants familiaux, bénévoles, nouvelles recrues), ne posséderaient pas. Du fait de l'ancrage naturel de ces compétences, elles seraient justement impossibles à transmettre. La notion de vocation permet donc de solidifier et défendre une identité professionnelle positive.

La vocation se définit également par l'ascèse, c'est-à-dire par l'investissement total de soi dans l'activité. Le don de soi et le désintéressement sont présentés par les aides-soignantes comme des marques de leur vocation (Sapiro 2007). Les aidessoignantes en gériatrie sont donc des acteurs professionnels qui revendiquent la possession depuis toujours d'une vocation à aider les personnes âgées, et à accepter les tâches qu'elles jugent rudes et parfois désagréables pour effectuer ce service. Ainsi, l'usage du terme de vocation permet aux aides-soignantes de mieux accepter la proximité à la souillure et la dureté des conditions de travail inhérentes au contexte organisationnel gériatrique (Marché-Paillé 2010).

Nous voyons donc en quoi la vocation est une construction identitaire qui a pour base une occultation des modalités de transmission des compétences professionnelles. Ce processus a deux significations précises. Il permet tout d'abord de se définir comme possesseur de compétences naturelles non transmissibles, afin de se construire une identité forte d'acteur professionnel compétent. Il permet également d'accepter plus facilement la dure réalité du travail en gériatrie. Nous allons montrer maintenant que les aides-soignantes n'hésitent pas à reconstruire une mémoire familiale et à se positionner dans l'ordre intergénérationnel de leur famille afin de mieux expliquer l'avènement de ce sentiment vocationnel.

\section{Une position d'endetté construite : une mémoire familiale reconstruite}

L'ensemble des acteurs que nous avons rencontrés tendent à présenter une image spécifique de la transmission de leurs compétences axée sur la naturalisation, et donc sur un oubli des modalités de leur transmission. Stroobants a théorisé ce processus de naturalisation dans le processus d'habilitation ; pour cet auteur, «un savoir-faire tacite est un savoir qui ne connaît plus les formes par lesquelles il a pu s'exprimer » (Stroobants 1993 : 17). Les acteurs en viennent à penser, en travaillant, qu'ils possèdent depuis toujours les compétences nécessaires pour faire le métier. En devenant habile, c'est à dire en acquérant les compétences transmises par la forma- 
tion et par l'expérience, les acteurs tendent parallèlement à oublier le processus par lequel ces compétences leur ont été transmises : «L'habileté transforme si radicalement le sujet de l'expérience qu'il ne sait plus ce qu'il a gagné en savoirs ni comment il y est parvenu » (Stroobants 1993 : 311). L'usage de la notion de vocation participe au processus d'habilitation.

Pour les aides-soignantes, la naturalisation des compétences prend sens dans le cadre d'un positionnement de soi au sein d'un ordre intergénérationnel. Elles disent en effet que leur vocation prend sens par le devoir moral qu'elles ressentent de rembourser une dette préalablement contractée avec leurs ascendants. Elles explicitent l'origine de cet héritage. Il s'agit de l'aide qui leur fut donnée par leurs parents ou leurs grands-parents lors de leur enfance. L'aide prend ici une définition très générale et ne se restreint pas à une liste d'actes ou de pratiques. Les aides-soignantes estiment devoir s'occuper des aînés comme, auparavant, des membres féminins de leur lignée se sont occupés d'elles.

- Moi, j'ai le respect de la personne âgée ; c'est déjà quelque chose que j'avais par mes origines, mais c'est vrai que j'ai appris beaucoup en arrivant ici. Aussi bien au niveau de l'intimité de la personne, j'ai appris énormément ici. Et, par le fait d'avoir perdu mes parents jeunes, j'essaie d'apporter à ces personnes âgées ce que je n'ai pas pu apporter aux miens alors qu'ils se sont beaucoup occupés de moi. Ça me paraît quelque chose de normal. (E2 - France)

- Ce sont mes grands-parents qui m'ont élevé puisque mes parents se sont séparés. J'étais très jeune, j'avais 8 mois, ma maman m'avait abandonnée et c'est ma grand-mère qui m'a recueillie. Et c'est ma grand-mère qui m'a élevée jusqu'à ce que je me marie. J'ai perdu ma grand-mère, ça fait un moment, et puis c'est pour ça que je travaille dans le milieu des personnes âgées. Par rapport à ma grand-mère qui m'a élevée et qui a su bien m'élever. C'est comme un échange que je fais maintenant. (E26 - Québec)

Les aides-soignantes revendiquent cette position d'endettées pour mieux solidifier la signification de leur rhétorique de vocation. En effet, être endetté, c'est devoir rendre un don qu'on a préalablement reçu. Pour reprendre les propos de Godbout et Caillé (1991 : 32), « qualifions de don toute prestation de bien ou de service effectuée, sans garantie de retour, en vue de créer, nourrir ou recréer le lien social entre les personnes ». Prestataires de service envers les personnes âgées, elles seront poussées par les conditions du métier à se positionner dans une situation de donataire. Le recours au long-terme, et notamment la réappropriation de la mémoire familiale, leur permet d'identifier leur pratique professionnelle comme une obligation morale, illustrée par cette rhétorique de la vocation. Effectivement, « la mise en dette sollicite la mémoire » (Bloch, Buisson \& Mermet 1991 : 85). La reconstruction biographique, et l'identification de soi comme l'héritier d'un service reçu, permet aux aidessoignantes de se replacer volontairement dans une économie de la réciprocité. Reprenant les propos de Bloch, Buisson et Mermet (1991 : 85) : 
«L'idée est que la place dans l'histoire généalogique, le rapport à ses ascendants ne sont pas reçus une fois pour toutes, ce que laisse peut-être trop rapidement entendre la notion de filiation, mais sont manipulés, au gré des relations d'obligations successives qu'on engage avec les membres de sa lignée et de la lignée de son conjoint, ouvrant ainsi un jeu de la mémoire. »

La reconstruction du lien de filiation, notamment maternel, appliqué à une logique de dette à rendre, est à la base de la naturalisation des compétences professionnelles. Se dire endetté d'un don familial normalise une position de donateur à des membres non-familiaux, soit les personnes âgées dépendantes, et accentue la croyance en la possession de compétences acquises naturellement. Par cette mise en récit de la filiation (Muxel 2002), les aides-soignantes se construisent un sens biographique à leur choix professionnel. Elles se réapproprient leur trajectoire en lui donnant un sens lié à la vocation professionnelle utilisée comme rhétorique.

Pour les aides-soignantes françaises et québécoises, se présenter comme endetté détient une signification identitaire. En effet, ce travail de la mémoire, ce «découpage de la mémoire » (Zonabend 1999) ne va pas sans raisons. Il permet tout d'abord aux aides-soignantes d'accepter plus facilement la réalité organisationnelle difficile et les conditions de travail pénibles. Il donne la possibilité également de tracer une frontière entre ceux qui ont hérité et ceux qui n'ont rien reçu.

\section{Une rhétorique de valorisation pour accepter un métier difficile}

Nous avons vu que les aides-soignantes que nous avons interrogées utilisent une terminologie spécifique pour exprimer l'origine de leurs compétences, soit le terme de vocation. Mais le principe vocationnel ne va pas, dans la rhétorique des aidessoignantes, sans une conception spécifique de la dureté du travail. Comment penser l'injonction «il faut aimer ça » que nous retrouvons dans leurs propos ? Quelle définition peut-on donner à la locution «ça » ? Il s'agit tout d'abord de la nécessité imposée par autrui de dire son goût pour un travail qui n'a pas nécessairement beaucoup d'attraits. Il s'agit également de fermer les portes du métier à celles qui ne détiendraient pas de compétences suffisantes et adéquates pour effectuer un travail de qualité. Mais cette expression énonce aussi et surtout une injonction à «tenir » face aux conditions de travail difficiles.

Le «ça » se définit comme le tacite de leur pratique professionnelle. Il est plus aisément résumable en un objet extérieur qu'en une pratique exprimable. « Ça », c'est le cadre professionnel réunissant la souillure, la mort et les difficiles conditions de travail. Aimer «ça », c'est accepter les conditions de travail qui existent dans le métier d'aide-soignante et admettre que sa trajectoire se confond avec ce destin professionnel. Les aides-soignantes tentent ainsi de mettre une distance entre leurs pratiques valorisantes (le relationnel) et certaines caractéristiques des tâches liées au 
« travail sale ». Le métier serait envié et haï tout à la fois. On constate cette ambiguïté dans certaines expressions qu'elles emploient, telles que : « il le faut bien » ou « qui le ferait, sinon ? », qui réfèrent à une terminologie relevant du domaine du sacrificiel. Ces phrases renvoient à l'idée d'accepter soi-même la fatigue au travail. Les acteurs font alors passer pour un choix individuel la souffrance qu'ils subissent.

- Ah ! Ouais, il n'y a rien à faire. Moi, je pense que pour travailler dans des milieux comme ça, il faut avoir la vocation. Moi, dès que j'ai fait le métier en tant qu'aide-soignante, après la formation, qui m'a pris un an, là je me suis dit : Ouais, c'est ça, c'est le métier que je veux faire. C'est sûr qu'il faut aimer ca, parce que tout le monde ne le ferait pas. (E18 - France)

On retrouve l'idée chère à Stroobants (1993), selon laquelle, pour être un professionnel habile, il faut se représenter «être fait pour ça », tout comme un contenant doit correspondre avec son contenu. Le contenant, c'est-à-dire l'emploi, a besoin d'un contenu préalablement construit, soit l'aide-soignante se pensant naturellement poussée à effectuer ce travail. On voit précisément ici que l'habileté, selon Stroobants, consiste autant en un processus de transmission des compétences au travailleur qu'un processus d'occultation des modalités de transmission de ses compétences.

- C'est une vocation parce que ce n'est pas donné à tout le monde. Il y a des personnes qui ne sont pas restées. Elles ne supportaient pas la personne âgée. Elles... Ne serait-ce que la toilette, un crachat, ou pour manger; il y a des personnes qui ne peuvent plus manger toutes seules et puis qui bavent. Il y a beaucoup de choses qui peuvent faire en sorte de te dégoûter. (E43 - Québec)

Ainsi, l'usage du terme de vocation permet aux aides-soignantes de penser qu'elles sont capables de «tenir » face à la complexité des tâches, en transformant une souffrance en une fierté personnelle :

- Moi, c'est plus par vocation que je fais ça. Donc, après, oui, il en faut, des gens comme nous. Après, oui, je suis fière de ce que je fais parce qu'il n'y a pas beaucoup de gens qui pourraient le faire. Moi, je pense qu'après, il faut être faite pour ce métier-là. Oui, ça doit être une vocation, tu ne peux pas faire ça comme ça. (E7 - France)

- Oui, je crois que c'est une vocation ; c'est une vocation, sinon... Pour aimer le travail, faut que ce soit une vocation. Moi, j'ai toujours aimé ça, je n'ai jamais eu de doute là-dessus ; il faut qu'on le travaille pour le savoir. (E9 - France)

La vocation devient alors la preuve de la capacité des acteurs à travailler malgré les difficiles conditions de travail. Avoir la vocation, c'est être poussé par une force invisible vers ce destin professionnel. En somme, l'usage de la vocation poursuit un but qui est bien davantage structurel qu'affinitaire : les aides-soignantes doivent reconstruire rapidement leur représentation de leur trajectoire afin de mieux accepter les difficiles conditions de travail. D'ailleurs, les sacrifices auxquels elles doivent consentir sont suffisamment grands pour que ces dernières ne souhaitent pas transmettre leur métier à leurs enfants. Notons que ce fait est valable également pour les 
deux contextes, français et québécois. Ce refus de conseiller les enfants à choisir le métier d'aide-soignante peut sembler quelque peu paradoxal lorsque l'on entend les acteurs définir de manière positive leur métier. Mais la naturalisation des compétences professionnelles permet aux aides-soignantes de mettre en place une double stratégie. D'un côté, elles peuvent se considérer elles-mêmes comme étant naturellement portées à faire ce métier, et ce, en procédant à une reconfiguration de leur trajectoire biographique. D'un autre côté, elles peuvent empêcher leurs enfants de faire le même métier en jugeant que ceux-ci ne détiennent ni les compétences professionnelles suffisantes, ni une vocation à choisir ce métier.

\section{Conclusion}

Nous avons montré dans cet article que l'usage du concept de mémoire dans le champ de la sociologie des professions est tout à fait approprié. En effet, la naturalisation des compétences et l'usage de la notion de vocation sont nécessaires pour les aides-soignantes autant françaises que québécoises, car cela leur permet d'accepter plus facilement leur contexte de travail et leur identité professionnelle malgré les tâches liées au «sale travail » qu'elles doivent réaliser dans les organisations gériatriques (souillure des personnes âgées, mort, etc.), et face aux difficiles conditions de travail (manque de personnel, surcharge, etc.). Ainsi, la reconstruction de la mémoire concernant leur trajectoire personnelle et professionnelle permet de solidifier un processus de subjectivation nécessaire qui leur permet d'accepter le difficile métier d'aide-soignante et d'y rester. Nous émettons l'hypothèse selon laquelle les aides-soignantes qui ne parviennent pas à réaliser cette naturalisation sont précisément celles qui parviennent le moins à supporter les conditions de travail inhérentes aux organisations gériatriques françaises et québécoises.

\section{Bibliographie}

Arborio, A.-M. (2001). Un personnel invisible: les aides-soignantes à l'hôpital. Paris: Anthropos.

Aubry, F. (2010). «La place du groupe de pairs dans l'intégration des nouvelles aides-soignantes dans les EHPAD ». SociologieS [en ligne], Premiers textes. Consultable en ligne : <http://sociologies.revues.org/index3291.html>

Bloch, F., Buisson M. \& Mermet, J-C. (1991). «Filiation, obligation familiale, continuité et discontinuité conjugale». In B. Bawin-Legros \& J. Kellerhalls (Dir.). Relations intergénérationnelles. Parenté - transmission - mémoire, Université de Genève/Université de Liège, pp. 83-92. 
Clément, S. \& Lavoie, J-P. (2005). Prendre soin d'un proche âgé. Les enseignements de la France et du Québec. Paris: Erès.

Glaser, B.-G. \& Strauss, A-L. (1967). The discovery of grounded theory. Strategies for qualitative research. Chicago: Aldine.

Godbout, J.-T. (2000). Le don, la dette et l'identité. Montréal: La découverte.

Godbout, J.-T. \& Caillé, A. (1991). «Le don existe-t-il (encore) ? . Revue du MAUSS, 11(1), pp. 11-32.

Grenier, N. (2008). Portrait de la main d'œuvre Préposés aux bénéficiaires (Rapport Grenier). Montréal: Ministère de la Santé et des Services sociaux du Québec.

Jacques-Jouvenot, D. (1997). Choix du successeur et transmission patrimoniale. Paris: L'harmattan.

Jacques-Jouvenot, D. (2007). «La place de l'élu dans la transmission professionnelle ». In C. Dardy, \& C. Fretigne (Dir.). L'expérience professionnelle et personnelle en question. Paris: L'harmattan, pp. 155-167.

Marché-Paillé, A. (2010). «Le dégoût dans le travail d'assistance aux soins personnels, s'en défendre mais pas trop », Travailler . 24(2), pp. 35-54.

Muxel, A. (2002). Individu et mémoire familiale. Paris: Nathan.

Sainsaulieu, I. (2003). Le malaise des soignants. Le travail sous pression à l'hôpital. Paris: L'Harmattan.

Sapiro, G. (2007). «La vocation artistique entre don et don de soi », Actes de la recherche en sciences sociales. 168(3), 4-11.

Stroobants, M. (1993). Savoir-faire et compétences au travail. Une sociologie de la fabrication des aptitudes. Bruxelles: Editions de l'Université de Bruxelles.

Suaud, C. (1975). «L'imposition de la vocation sacerdotale », Actes de la recherche en sciences sociales. 3 , pp. 2-17.

Zonabend, F. (1999). La mémoire longue. Paris: Jean-Michel Place. 